\title{
PgFur participates differentially in expression of virulence factors in more virulent A7436 and less virulent ATCC 33277 Porphyromonas gingivalis strains
}

\author{
Michał Śmiga, Paulina Stępień, Mariusz Olczak and Teresa Olczak *i]
}

\begin{abstract}
Background: Porphyromonas gingivalis is considered a keystone pathogen responsible for chronic periodontitis. Although several virulence factors produced by this bacterium are quite well characterized, very little is known about regulatory mechanisms that allow different strains of $P$. gingivalis to efficiently survive in the hostile environment of the oral cavity, a typical habitat characterized by low iron and heme concentrations. The aim of this study was to characterize P. gingivalis Fur homolog (PgFur) in terms of its role in production of virulence factors in more (A7436) and less (ATCC 33277) virulent strains.
\end{abstract}

Results: Expression of a pgfur depends on the growth phase and iron/heme concentration. To better understand the role played by the PgFur protein in P. gingivalis virulence under low- and high-iron/heme conditions, a pgfur-deficient ATCC 33277 strain (TO16) was constructed and its phenotype compared with that of a pgfur A7436-derived mutant strain (TO6). In contrast to the TO6 strain, the TO16 strain did not differ in the growth rate and hemolytic activity compared with the ATCC 33277 strain. However, both mutant strains were more sensitive to oxidative stress and they demonstrated changes in the production of lysine- (Kgp) and arginine-specific (Rgp) gingipains. In contrast to the wildtype strains, TO6 and TO16 mutant strains produced larger amounts of HmuY protein under high iron/heme conditions. We also demonstrated differences in production of glycoconjugates between the A7436 and ATCC 33277 strains and we found evidence that PgFur protein might regulate glycosylation process. Moreover, we revealed that PgFur protein plays a role in interactions with other periodontopathogens and is important for P. gingivalis infection of THP-1-derived macrophages and survival inside the cells. Deletion of the pgfur gene influences expression of many transcription factors, including two not yet characterized transcription factors from the Crp/Fnr family. We also observed lower expression of the CRISPR/Cas genes.

Conclusions: We show here for the first time that inactivation of the pgfur gene exerts a different influence on the phenotype of the A7436 and ATCC 33277 strains. Our findings further support the hypothesis that PgFur regulates expression of genes encoding surface virulence factors and/or genes involved in their maturation.

Keywords: Porphyromonas gingivalis, Ferric uptake regulator, PgFur, Iron, Heme, Virulence

\footnotetext{
* Correspondence: teresa.olczak@uwr.edu.pl

Laboratory of Medical Biology, Faculty of Biotechnology, University of

Wrocław, F. Joliot-Curie 14A St, 50-383 Wrocław, Poland
}

(c) The Author(s). 2019 Open Access This article is distributed under the terms of the Creative Commons Attribution 4.0 International License (http://creativecommons.org/licenses/by/4.0/), which permits unrestricted use, distribution, and reproduction in any medium, provided you give appropriate credit to the original author(s) and the source, provide a link to the Creative Commons license, and indicate if changes were made. The Creative Commons Public Domain Dedication waiver (http://creativecommons.org/publicdomain/zero/1.0/) applies to the data made available in this article, unless otherwise stated. 


\section{Background}

Periodontal diseases, the most common chronic infections in humans, are characterized by the loss of toothsupporting tissues $[1,2]$. Analyses of subgingival samples revealed the presence and relative abundance of periodontal pathogens, including the "red complex" bacteria (Porphyromonas gingivalis, Tannerella forsythia and Treponema denticola) [3], which are associated with the clinical features of chronic periodontitis [4, 5]. Other bacteria, such as Streptococcus gordonii, a member of the "yellow complex", and Prevotella intermedia, a member of the "orange complex", serve as early colonizers or bridging species to members of the "red complex" [3, 6, 7]. Among the putative periodontopathogens, $P$. gingivalis is considered the main etiologic agent and a keystone pathogen responsible for initiation and progression of chronic periodontitis $[8,9]$. $P$. gingivalis is a constituent of a mixed-species biofilm of the oral cavity [10,11]; however, it can also enter gingival epithelial and immune cells, and can spread among host cells, thus contributing to its survival in the oral cavity [12-16].

$P$. gingivalis is a heme auxotroph and uptake of this compound is required as an iron and protoporphyrin IX source, as well as being crucial for survival and efficient establishment of infection by this bacterium. Among well-characterized heme acquisition systems of $P$. gingivalis is that encoded by the $h m u$ operon, comprising HmuR, a typical TonB-dependent receptor involved in heme transport through the outer membrane, HmuY, a heme-binding hemophore-like protein exhibiting unique structure and heme-binding properties, and four proteins with, as yet, unknown function [17]. P. gingivalis produces higher levels of $\mathrm{HmuY}$ when the bacterium grows under low-iron/heme conditions or as a biofilm constituent $[18,19]$, as well as intracellularly in host cells [16]. The HmuY protein is associated with both the bacterial outer membrane and outer-membrane vesicles (OMVs) through a lipid anchor $[19,20]$, and can also be shed as an intact, soluble protein as a result of the limited proteolytic processing by $P$. gingivalis lysine-specific gingipain K (Kgp) [20, 21]. Heme can also be acquired from host hemoproteins through the high proteolytic activity of $P$. gingivalis, caused mainly by a group of cysteine proteases, comprising secreted and membraneassociated gingipains [22, 23]. Arginine-specific gingipains (RgpA and $\operatorname{RgpB}$ ) are encoded by the $\operatorname{rgp} A$ and $r g p B$ genes, and Kgp by the kgp gene. The predominant form of Kgp and RgpA is a complex of a catalytic domain with hemagglutinin/adhesion domains.

Although several mechanisms of $P$. gingivalis virulence, including uptake of iron, heme, and other nutrients are quite well characterized [17, 23], very little is known about regulatory mechanisms that allow $P$. gingivalis to survive in the very hostile environment of the oral cavity, a typical habitat characterized by low iron and heme availability. Regulation of gene expression in bacteria is usually carried out at the transcriptional level by DNA-binding proteins and at the post-transcriptional level by protein-protein interactions [24]. Among the best characterized bacterial regulators, there is a ferric uptake regulator (Fur), a member of the FUR superfamily, comprising a variety of metalloregulators, which can regulate expression of genes at the transcriptional level in response to environmental factors through several different mechanisms $[25,26]$. In the classical Fur regulation paradigm, under high-iron conditions Fur becomes ferreted and binds to specific "Fur box" sequences located in promoters of regulated genes, thereby preventing their transcription through steric hindrance of RNA polymerase. Under iron-restricted conditions, the Fur- $\mathrm{Fe}^{2+}$ complex dissociates and the repressor becomes inactive, which leads to the transcription of target genes. In contrast to iron-dependent DNA binding activity, Fur in some systems can sense signals other than iron, including heme, or bind to DNA in the absence of a metal cofactor [27-29]. Importantly, it has been well documented that Fur functions as a global regulator that controls not only the expression of iron acquisition systems, but also a large number of genes involved in different cellular processes, such as stress response and production of virulence factors [25, 26, 30].

P. gingivalis possesses a single predicted Fur homolog, which we termed PgFur [31]. Our recent data suggested that iron and heme limitation together with inactivation of the pgfur gene affects directly or indirectly the expression of several genes encoding important virulence factors of P. gingivalis A7436 strain. According to our hypothesis, $P$. gingivalis employs PgFur to initiate a complex, multilayer network used to regulate virulence in the host iron/heme-limited environment. To date, several genomes of $P$. gingivalis strains obtained from patients have been sequenced and deposited in databases. These strains differ in both the genotype and the exhibited phenotype. Therefore, the aim of this study was to characterize the importance of $P$. gingivalis PgFur in the production of virulence factors in more (A7436) and less (ATCC 33277) virulent strains.

\section{Methods}

\section{Bacterial strains and growth conditions}

P. gingivalis wild-type (A7436, ATCC 33277), pgfur mutant (TO6, TO16), complemented mutant (TO6 + pgfur, TO16 + pgfur), and control (TO6 + pTIO-tetQ, TO16 + pTIO-tetQ) strains were grown anaerobically at $37^{\circ} \mathrm{C}$ for 3-10 days on blood agar plates (ABA) (Biocorp) as described previously $[18,31]$. These cultures were used as the inoculum for growth in liquid basal medium (BM) comprising 3\% trypticase soy broth (Biocorp), containing 
$0.5 \%$ yeast extract (Biocorp), $0.05 \%$ cysteine (Sigma), 0.5 $\mathrm{mg} / \mathrm{l}$ menadione (Fluka), and supplemented with $7.7 \mu \mathrm{M}$ hemin $(\mathrm{Hm})$ or $0.1-1.0 \mathrm{mM} \mathrm{ZnCl}_{2}(\mathrm{Zn})$. To mimic lowiron/heme conditions, bacteria were grown in $\mathrm{BM}$ in the absence of heme and in the presence of an iron chelator, $160 \mu \mathrm{M}$ 2,2-dipyridyl (DIP) (Sigma) or $0.5 \mathrm{mM}$ EDTA (EDTA) (Sigma). To examine the influence of oxidative stress, bacteria were grown in $\mathrm{BM}$ lacking cysteine and supplemented with 0.05 or $0.25 \mathrm{mM} \mathrm{H}_{2} \mathrm{O}_{2}$. TO6 and TO16 strains were maintained in the presence of $1 \mu \mathrm{g} /$ $\mathrm{ml}$ erythromycin, whereas complemented mutant (TO6 + pgfur, TO16 + pgfur) and control (TO6 + pTIOtetQ, TO16 + pTIO-tetQ) strains in the presence of $1 \mu \mathrm{g} / \mathrm{ml}$ erythromycin and $1 \mu \mathrm{g} / \mathrm{ml}$ tetracycline. Bacterial growth in liquid culture media was determined by measuring optical density at $600 \mathrm{~nm}\left(\mathrm{OD}_{600}\right)$ and on $\mathrm{ABA}$ plates by visual inspection.

\section{Construction of $P$. gingivalis pgfur mutant, complemented} mutant and control strains

Inactivation of the $P$. gingivalis pgfur gene (PGA7_ 00014570) in the A7436 wild-type strain was previously reported [31], resulting in the TO6 mutant strain. The $P$. gingivalis pgfur gene in the wild-type ATCC 33277 strain (PGN_1503) was analogously inactivated in this study by introducing the ermF cassette (encoding the erythromycin resistance gene from Bacteroides fragilis) to yield a pgfur-ermF-pgfur DNA fragment, resulting in the TO16 mutant strain. In addition, complemented mutant (TO6 + pgfur, TO16 + pgfur) and control (TO6 + pTIOtetQ, TO16 + pTIO-tetQ) strains were constructed using the modified pTIO-1 vector, originally designed and constructed by Tagawa et al. [32], for electroporation of $P$. gingivalis. Briefly, the ermF cassette in the original pTIO-1 plasmid was first replaced by the tetQ cassette from $B$. thetaiotaomicron (encoding the tetracycline resistance gene), yielding the pTIO-tetQ plasmid. This modified plasmid was introduced into TO6 and TO16 mutant strains to construct control TO6 + pTIO-tetQ and $\mathrm{TO} 16+$ pTIO-tetQ strains. To construct complemented mutant strains, the pgfur gene with the original promoter region was introduced into XhoI and BamHI restriction sites of the pTIO-tetQ plasmid. All primers used in this study are listed in Additional file 1: Table S1. P. gingivalis strains were electroporated with appropriate plasmids and subsequently grown as described previously [31, 32]. All constructs and homologous recombinations between the constructs and the chromosomal DNA of $P$. gingivalis were verified by PCR and sequencing analyses (data not shown).

\section{Co-aggregation assay}

P. gingivalis, T. forsythia ATCC 43037 and P. intermedia 17 were grown under anaerobic conditions as described previously [33]. Streptococcus gordonii ATCC 10558 was grown in 5\% Tryptic soy broth (TSB, Becton Dickinson) under a $\mathrm{CO}_{2}$-enriched atmosphere (Thermo Scientific). To examine the co-aggregation ability of $P$. gingivalis wild-type and pgfur mutant strains with other species, bacteria were incubated anaerobically for $6 \mathrm{~h}$ in $20 \mathrm{mM}$ phosphate buffer, pH 7.4, containing $140 \mathrm{mM} \mathrm{NaCl}$ (PBS). The co-aggregation ability was determined by measuring the decrease in OD at $600 \mathrm{~nm}$.

\section{Microarray analysis}

Genome-wide expression profiling was carried out (IMGM, Martinsried, Germany) using $P$. gingivalis wildtype (A7436 and ATCC 33277) and pgfur mutant (TO6 and TO16) strains, grown under low- (DIP) or highiron/heme $(\mathrm{Hm})$ conditions analogously as described previously for $P$. gingivalis A7436 wild-type and TO6 mutant strains [31].

\section{Quantitative reverse transcriptase-polymerase chain reaction (qRT-PCR)}

RNA was extracted from $0.5 \times 10^{8}-4 \times 10^{8} P$. gingivalis cells using the Total RNA Mini Kit (A\&A Biotechnology). Isolated RNA was treated with DNase I (A\&A Biotechnology) and purified using the Clean-Up RNA Concentrator Kit (A\&A Biotechnology). RNA integrity was verified using a spectrophotometric method and separating RNA samples on an agarose gel. Reverse transcription was carried out using $1 \mu \mathrm{g}$ of RNA and the SensiFAST cDNA Synthesis Kit (Bioline).

PCR was carried out using the SensiFAST SYBR NoROX Kit (Bioline) and the LightCycler 96 System (Roche). The amplification reaction comprised initial denaturation at $95^{\circ} \mathrm{C}$ for $2 \mathrm{~min}, 40$ cycles of denaturation at $95^{\circ} \mathrm{C}$ for $5 \mathrm{~s}$, primer annealing at $60^{\circ} \mathrm{C}$ for $10 \mathrm{~s}$, and extension at $72{ }^{\circ} \mathrm{C}$ for $20 \mathrm{~s}$. The melting curves were analyzed to monitor the quality of PCR products. Relative quantification of expression of respective genes was determined in comparison with the $16 S$ rRNA gene of $P$. gingivalis (PGA7_00000960 for A7436 and PGN_r0001 for ATCC 33277) as a reference, using the Pfaffl method [34]. All samples were run in triplicate for the target and reference genes, in three independent experiments. Notemplate controls were included on each reaction plate to check for contamination. Negative controls consisting of untranscribed RNA (no-RT controls) were performed to check for genomic DNA contamination. All primers used in this study are listed in Additional file 1: Table S1.

\section{Sodium dodecyl sulfate-polyacrylamide gel electrophoresis (SDS-PAGE) and Western blotting}

Proteins were prepared by sample incubation with Laemmli loading buffer $\left(95^{\circ} \mathrm{C}, 10 \mathrm{~min}, 1200 \mathrm{rpm}\right)$ and 
separated in $12 \%$ polyacrylamide gels using SDS-PAGE as described previously [31]. The protein amount of cell lysate loaded per well was normalized against $P$. gingivalis culture volume and the number of cells determined by measuring $\mathrm{OD}$ at $600 \mathrm{~nm}$. Proteins were either visualized by Coomassie Brilliant Blue G-250 (CBB G-250) staining or transferred onto nitrocellulose membranes (Millipore). HmuY protein was detected with anti-HmuY antibodies as described previously [33].

\section{Lectin assay}

An equivalent of $\mathrm{OD}_{600}=0.3$ of bacterial cultures was separated using SDS-PAGE and transferred onto nitrocellulose membranes. After blocking with Carbo-Free Blocking Solution (Vector Laboratories), membranes were incubated with biotinylated lectins $(1: 3300$; Vector Laboratories) in $50 \mathrm{mM}$ Tris $-\mathrm{HCl}, \mathrm{pH} 7.5$, containing $150 \mathrm{mM} \mathrm{NaCl}$ (TBS), $0.2 \%$ Tween-20, $1 \mathrm{mM} \mathrm{CaCl}_{2}$ and $1 \mathrm{mM} \mathrm{MnCl} 2$. Lectin binding was detected using alkaline phosphatase-conjugated avidin D (Vector Laboratories) and visualized with NBT/BCIP substrate solution (Roche). Equal protein loading was determined by staining proteins in gels using CBB G-250 or on a nitrocellulose membrane by staining with Ponceau $S$.

\section{Bacterial cell fractionation}

Bacterial cultures were centrifuged at $20,000 \times g$ for 20 $\min$ at $4{ }^{\circ} \mathrm{C}$. Bacterial pellets were washed twice with PBS and used to analyze the whole cell fraction (WC). The supernatant was filtered using $0.22 \mu \mathrm{m}$ filters to obtain the cell-free fraction and subsequently centrifuged at $100,000 \times g$ for $2 \mathrm{~h}$ at $4{ }^{\circ} \mathrm{C}$ to separate the outermembrane vesicles (OMV) fraction. The soluble fraction (cell-free supernatant; CM) was concentrated $25 \times$ using Amicon Ultra-4 Centrifugal Filter Ultracel-10 K units (Millipore).

\section{Determination of proteolytic activity}

Whole $P$. gingivalis cultures or cell-free culture media were examined in order to determine proteolytic activity. The total proteolytic activity was measured using azocasein protease substrate according to the manufacturer's protocol (Sigma). To analyze proteolytic activity specific for Arg- and Lys-specific gingipains, No-benzoyl-DL-arginine 4-nitroanilide hydrochloride (BApNA) (Sigma) and $\mathrm{N}$-(p-tosyl)-Gly-Pro-Lys 4-nitroanilide acetate salt (Sigma) were used, respectively [35].

\section{THP-1 cultures and infection assay}

Human acute monocytic leukemia cells (THP-1) were grown in RPMI-1640 medium (Biowest), supplemented with $10 \%$ heat-inactivated fetal bovine serum (FBS, Cytogen), $2 \mathrm{mM}$ L-glutamine (Cytogen), $100 \mathrm{U} / \mathrm{ml}$ penicillin and $100 \mu \mathrm{g} / \mathrm{ml}$ streptomycin (Cytogen) in a humidified atmosphere of $95 \%$ air and $5 \% \mathrm{CO}_{2}$ at $37^{\circ} \mathrm{C}$ in a $\mathrm{CO}_{2}$ incubator (Sanlab). THP-1 cells were seeded at $1.0 \times 10^{6} \%$ $\mathrm{ml}$ in 12-well plates and differentiated toward macrophages by treatment with $0.01 \mu \mathrm{g} / \mathrm{ml}$ phorbol 12 myristate 13-acetate (PMA, Sigma Aldrich) for $48 \mathrm{~h}$, as described previously [36].

Live $P$. gingivalis wild-type and pgfur mutant strains were grown to the early stationary phase in $\mathrm{BM}+\mathrm{Hm}$, pelleted by centrifugation $\left(4000 \times g, 20 \mathrm{~min}\right.$ at $\left.4{ }^{\circ} \mathrm{C}\right)$, and washed twice with PBS. Bacteria were added to THP-1derived macrophages cultured without antibiotics in 12well plates at a multiplicity of infection (MOI) of 100 and incubated for $4 \mathrm{~h}$ at $37^{\circ} \mathrm{C}$ as described previously [31]. Then, the cells were washed 3 times with PBS and one portion out of four portions was lysed with water to determine bacteria attached to and present inside the host cells. To the remaining samples, fresh RPMI 1640 medium was added, supplemented with $300 \mu \mathrm{g} / \mathrm{ml}$ gentamicin and $200 \mu \mathrm{g} / \mathrm{ml}$ metronidazole. After $1 \mathrm{~h}$, one cell portion was washed 3 times with PBS and lysed to determine the amount of live cells present inside the host cells. After 8 and $24 \mathrm{~h}$, the two last cell portions were washed 3 times with PBS and lysed to determine live bacteria present inside the host cells. Serial dilutions of samples were plated on blood agar plates and colony forming units per $\mathrm{ml}$ $(\mathrm{CFU} / \mathrm{ml})$ were determined. All experiments were performed three times in duplicate.

\section{Results}

In our previous study, we reported preliminary analysis of a pgfur mutant strain (TO6) constructed in the more virulent A7436 wild-type strain [31] and suggested that PgFur can influence in vivo $P$. gingivalis growth and virulence, allowing efficient infection through a complex regulatory network. To provide more accurate and deeper understanding of the biological role played by PgFur in the production of virulence factors, we inactivated the $P$. gingivalis pgfur gene also in the less virulent ATCC 33277 strain. Complementation of the mutant strains with the native pgfur gene resulted in partial reversion of the mutants' phenotypes (Additional file 2: Fig. S1a and data not shown). We also demonstrated that introduction of the pTIO-tetQ plasmid alone to the mutant strains did not change control strains' phenotypes (data not shown).

\section{Expression of pgfur gene}

Determination of the pgfur transcript in both $P$. gingivalis wild-type strains showed differences when low-iron/ heme (DIP) conditions were compared at the indicated time points with high-iron/heme $(\mathrm{Hm})$ conditions (Fig. 1 a). In general, our results demonstrated that in both strains mRNA encoding PgFur protein is produced at higher levels after prolonged growth $(24 \mathrm{~h})$ when 


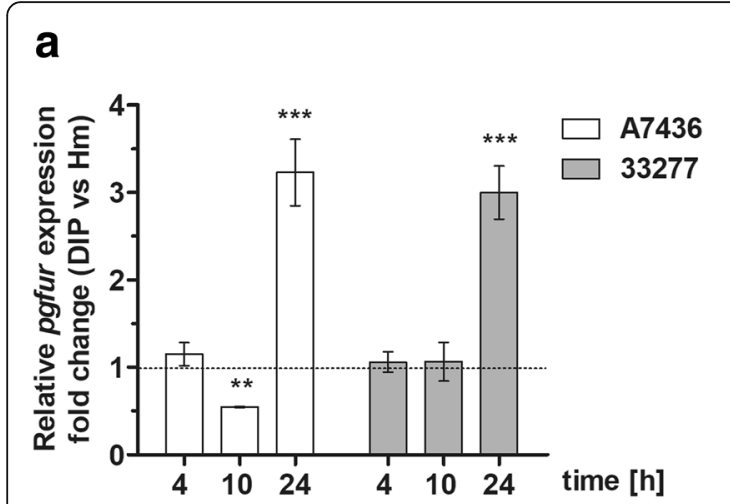

b

Fig. 1 Expression of pgfur gene in $P$. gingivalis strains. Relative expression of the pgfur gene was determined using RT-qPCR and shown in relation to iron/heme availability $(\mathbf{a})$ and additionally to growth phase $(\mathbf{b})$ at the indicated time points. Data are shown as relative levels of the mRNA normalized to $16 \mathrm{~S} r R N A$ mRNA and presented as mean \pm standard deviation (mean \pm SD) from three replicates. ${ }^{* *} P<0.01,{ }^{* * *} P<0.001$

bacteria grow under low iron/heme conditions. Interestingly, the A7436 strain under these conditions exhibited unusual decreased pgfur gene expression after $10 \mathrm{~h}$.

Further we analyzed influence of the growth phase on pgfur gene expression by comparing its expression determined after 10 and $24 \mathrm{~h}$ with the expression determined after $4 \mathrm{~h}$ in bacteria grown under low- or high-iron/ heme conditions. The highest pgfur expression was observed at the early growth phase for both A7436 and ATCC 33277 strains (Fig. 1 b). This effect was more pronounced when bacteria grew under low-iron/heme conditions, and when bacterial cultures at early stationary phase were compared with the growth at the early stage of the cultures. This would suggest that culture density might influence expression of the pgfur gene.

\section{Phenotypic characterization of pgfur mutant strains}

Characterization of phenotypes of the pgfur mutant strains revealed different morphological and physiological effects of the gene inactivation in the two $P$. gingivalis strains. In accordance with our previous studies [31], growth of the TO6 mutant strain in liquid media under high-iron/heme conditions $(\mathrm{Hm})$ resulted in higher cell density compared with the A7436 wild-type strain (Fig. 2 a). In contrast, the TO16 mutant strain did not differ significantly in growth ability in liquid media under these conditions compared with the ATCC 33277 wild-type strain (Fig. 2 b). These findings did not correlate with the luxR gene expression, one of the components responding to bacterial culture density (Additional file 3: Table S2). To produce iron/heme-depleted conditions, dipyridyl (DIP) was used and heme was not added to the basal medium. No significant differences in growth ability in the case of all examined strains were observed under these conditions (Fig. $2 \mathrm{a}$ and b). To analyze whether other metals, such as zinc, may affect $P$. gingivalis growth, EDTA was used. This compound was less effective in ion chelation, resulting in better $P$. gingivalis growth compared with DIP. Also under these conditions, no significant differences were found between the strains examined (data not shown). In addition, $P$. gingivalis growth in the presence of zinc excess ( $\mathrm{Zn}$ ) was analyzed. As expected, these conditions caused growth inhibition; however, both wild-type and mutant strains were able to recover and restart replication in the presence of up to $1 \mathrm{mM} \mathrm{ZnCl}$ (data not shown). The only difference was significantly longer time required for growth recovery of ATCC 33277 and TO16 strains (e.g., $\sim 24 \mathrm{~h}$ in the presence of $0.5 \mathrm{mM}$ $\mathrm{ZnCl}_{2}$ ) compared with $\mathrm{A} 7436$ and TO6 strains (e.g., 16 $\mathrm{h}$ in the presence of $0.5 \mathrm{mM} \mathrm{ZnCl}_{2}$ ).

When bacteria were grown on blood agar plates for a prolonged time, a significantly higher hemolytic activity (Fig. 2 c) and brighter colony color (Fig. 2 d) of the TO6 strain were observed compared with both wild-type strains and the TO16 mutant strain. When the ability of heme binding to the cell surface of whole live bacteria was examined, no significant differences, except lower heme binding in the case of the TO6 mutant strain, were demonstrated (Additional file 4: Figure S2). This might, at least in part, explain the lower colony pigmentation of the TO6 mutant cells (Fig. 2 d). Interestingly, the mutant TO16 strain exhibited different colony shape compared with the wild-type strain (Fig. 2 d).

Proteins belonging to Fur family may also control the expression of genes engaged in redox sensing [37]. Therefore, we examined pgfur mutant strains as regards their susceptibility to oxidative stress. Data shown in Fig. 3 demonstrated that both TO6 and TO16 mutant strains were more susceptible to oxidizing conditions. In addition, the ATCC 33277 wild-type strain was more susceptible to the negative effect of $\mathrm{H}_{2} \mathrm{O}_{2}$ compared with the A7436 wild-type strain. However, these findings did not correlate with $\operatorname{xy} R$ gene expression examined under different iron and heme availability (Additional file 4: Table S2). 

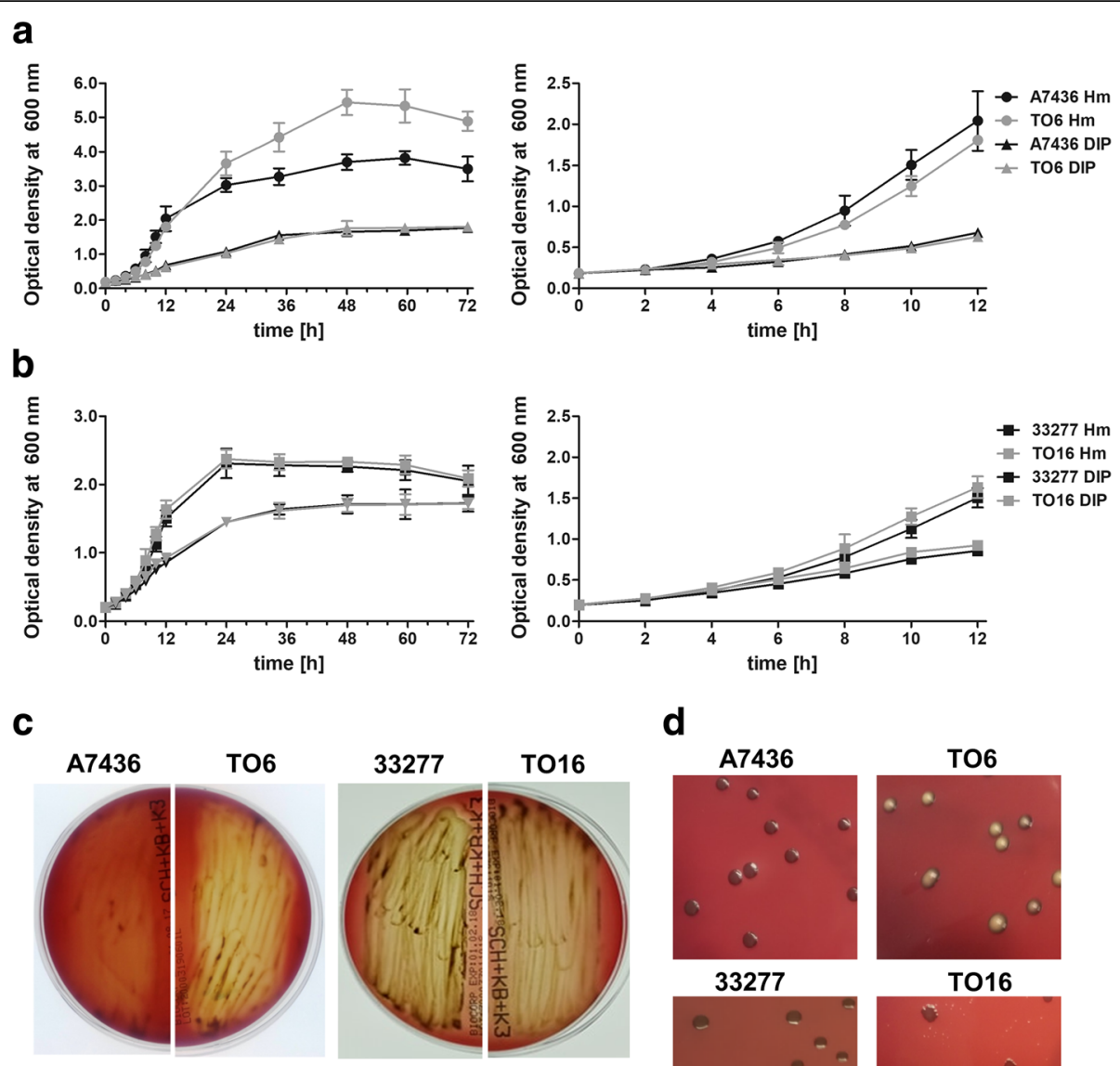

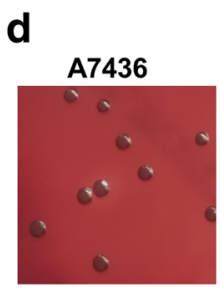

33277

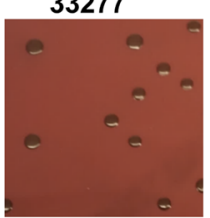

T06

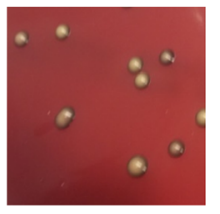

T016

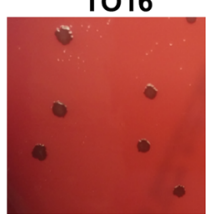

Fig. 2 Phenotypic characterization of $P$. gingivalis pgfur mutant strains. a, b P. gingivalis wild-type (A7436 and ATCC 33277) and pgfur mutant (TO6 and TO16) strains were grown in liquid medium. Bacterial cultures were started at $\mathrm{OD}_{600} \sim 0.2$ and the growth in basal medium supplemented with $7.7 \mu \mathrm{M}$ hemin $(\mathrm{Hm})$ or $160 \mu \mathrm{M}$ dipyridyl (DIP) was monitored by measuring optical density at $600 \mathrm{~nm}$ at the indicated time points. $\mathbf{c}$, $\mathbf{d}$ Growth of P. gingivalis wild-type (A7436 and ATCC 33277) and pgfur mutant (TO6 and TO16) strains on blood agar plates. Hemolysis (c) and colony color and shape (d) were visually observed for up to 10 days and pictures taken after 3 (c) and 5 (d) days, respectively are shown. Representative data out of 3 independent experiments performed in triplicate with a similar tendency are shown

To demonstrate the influence of the PgFur on interaction ability with other oral bacteria, co-aggregation between $P$. gingivalis and selected species was examined. As shown in Fig. 4 a and b (left panel), the TO6 mutant strain co-aggregated more efficiently with $T$. forsythia and $P$. intermedia, compared with the A7436 strain. Surprisingly, the TO16 mutant strain exhibited the opposite effect (Fig. $4 \mathrm{a}$ and b; right panel). No differences between the wild-type and pgfur mutant strains were observed when $P$. gingivalis and $S$. gordonii were examined (Fig. $4 \mathrm{c}$ ).

\section{Gene expression after pgfur inactivation}

To analyze expression of $P$. gingivalis genes upon $p g f u r$ inactivation on the global scale, we employed microarray analysis and compared the gene expression profiles between the wild-type and mutant strains grown in liquid iron and heme rich media $(\mathrm{Hm})$ or starved of iron and heme (DIP). Determination of gene expression in the TO16 mutant strain versus the ATCC 33277 wild-type strain showed changes in significantly lower number of genes and less significant changes in their expression levels compared with the TO6 mutant strain versus the A7436 wild-type strain, suggesting a smaller impact of PgFur on global gene expression in the ATCC 33277 strain. Therefore, we selected genes encoding potential virulence factors whose expression has been significantly changed in both $P$. gingivalis strains as examined by microarray analysis and determined their expression at various growth stages using RT-qPCR.

Among a variety of genes engaged in the regulation of virulence factors' expression are those encoding 

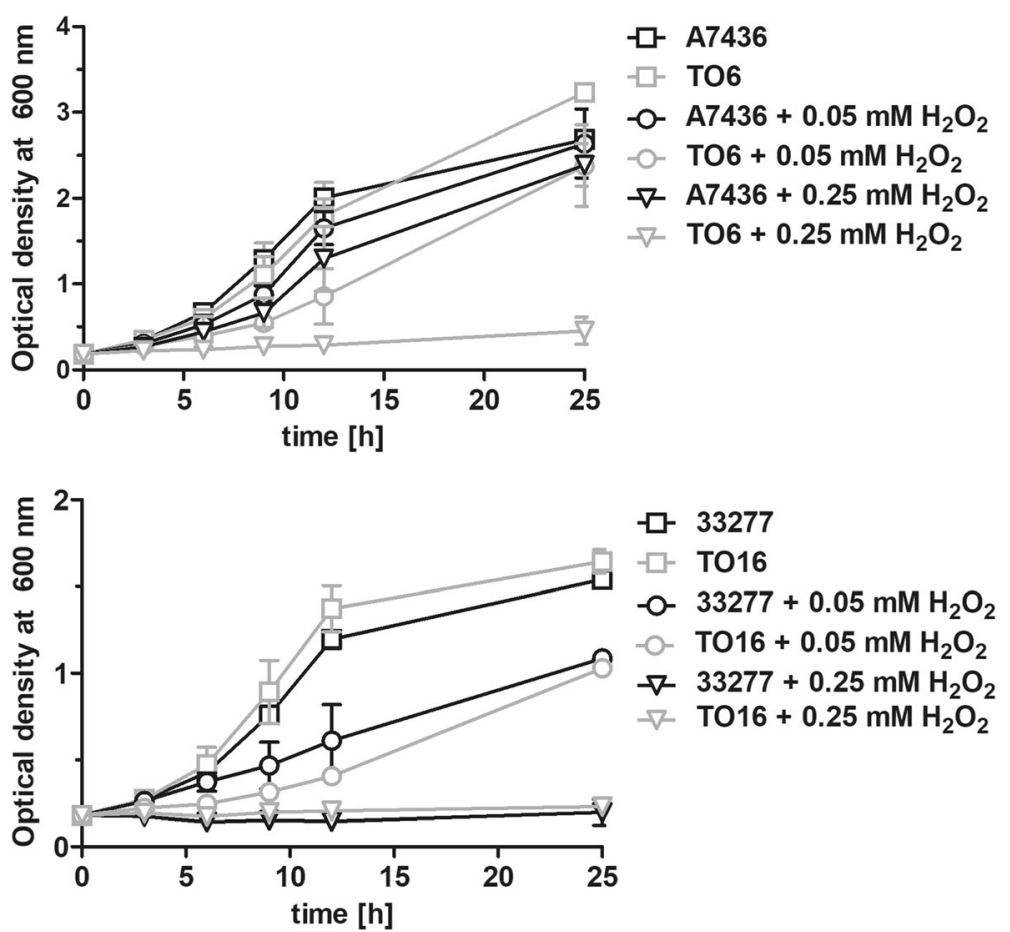

Fig. 3 P. gingivalis growth in a liquid medium in the presence of $\mathrm{H}_{2} \mathrm{O}_{2}$. Bacterial cultures were started at $\mathrm{OD}_{600} \sim 0.2$ and the growth in basal medium supplemented with $7.7 \mu \mathrm{M}$ hemin and $\mathrm{H}_{2} \mathrm{O}_{2}$ was monitored by measuring optical density at $600 \mathrm{~nm}$ at the indicated time points

potential transcription factors. We observed lower expression of PGA7_00015190/PGN_1569 and PGA7_ $00004090 / P G N \_0537$ genes, especially in the early stationary growth phase in both TO6 and TO16 mutant strains (Additional file 3: Table S2). Both genes encode putative transcription factors belonging to the Crp/Fnr superfamily. Surprisingly, the expression pattern of the PGA7_00009810/PGN_0970 gene encoding sigma factor differed in TO6 and TO16 mutant strains compared with the wild-type strains. Also worth noting are differences in expression of genes encoding proteins associated with the CRISPR/Cas system. Both TO6 and TO16 mutant strains demonstrated lower expression of these genes compared with the wild-type counterparts, especially during growth under high iron/heme conditions (Additional file 3: Table S2). We hypothesize that the above-mentioned proteins would participate in a multilayer regulatory network in collaboration with PgFur and also with other, so far unidentified proteins.

\section{Proteolytic activity of $P$. gingivalis}

Recently, we demonstrated that PgFur could be involved in the regulation of hemolytic/proteolytic activity of $P$. gingivalis [31]. Therefore, in this study, we first determined the total proteolytic activity of whole $P$. gingivalis cultures using azocasein as a protease substrate. When bacteria were grown under high-iron/heme conditions $(\mathrm{Hm})$, only the TO6 mutant strain exhibited significantly higher proteolytic activity compared with the A7436 wild-type strain (Fig. 5 a), corroborating data presented in Fig. 2c. Under low-iron/heme conditions (DIP), both TO6 and TO16 mutant strains exhibited lower proteolytic activity, but only the TO16 strain demonstrated statistically significant differences. Then, we determined gingipain activity of whole $P$. gingivalis cultures using substrates specific for Rgp and Kgp gingipains (Fig. 5 b). Both TO6 and TO16 mutant strains, with more pronounced differences observed in the case of the TO6 mutant strain, exhibited significantly lower Kgp activity under both high- (Hm) and low-iron/heme (DIP) conditions compared with the wild-type strains. Rgp activity was lower in both TO6 and TO16 mutant whole cultures under high-iron/heme $(\mathrm{Hm})$ conditions. In contrast, under low-iron/heme (DIP) conditions Rgp activity in whole bacterial cultures was higher in the case of both mutant strains compared with the wild-type counterparts. Further, we determined the activity of soluble gingipains secreted into the culture media (Fig. 6). Under high-iron/heme $(\mathrm{Hm})$ conditions, Kgp activity was significantly lower in the case of the TO6 mutant strain, whereas under low-iron/heme (DIP) conditions the TO16 mutant strain exhibited significantly higher Kgp activity. A similar pattern of Rgp activity was observed for both TO6 and TO16 mutant strains, being lower under high-iron/heme $(\mathrm{Hm})$ conditions and higher under low-iron/heme (DIP) conditions. These data are 

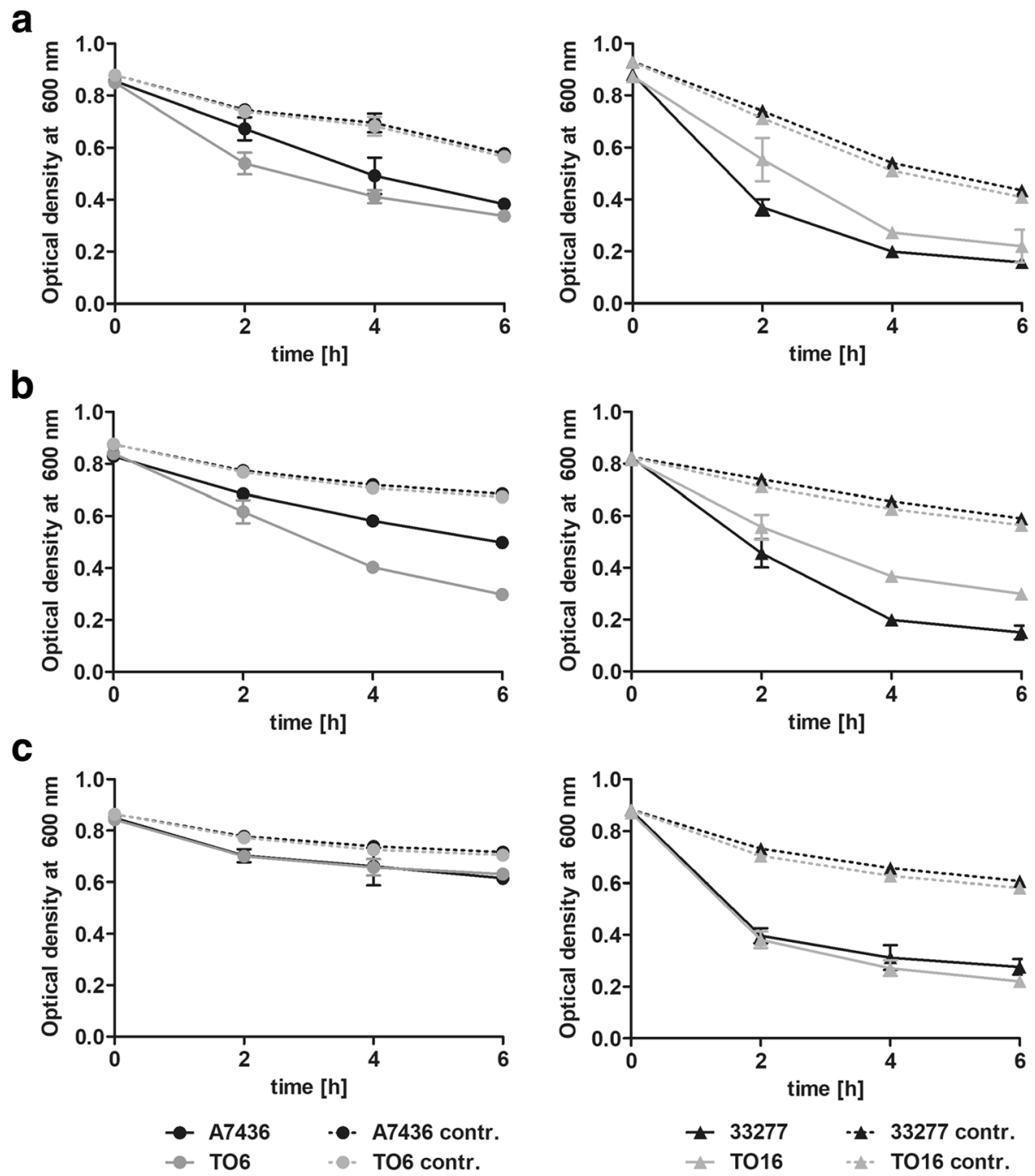

Fig. 4 Interaction of P. gingivalis wild-type (A7436 and ATCC 33277) and pgfur mutant (TO6 and TO16) strains with T. forsythia (a), P. intermedia (b), and S. gordonii (c). The tendency to co-aggregate was monitored by measuring optical density at $600 \mathrm{~nm}$ at the indicated time points. Control samples contain mono-species cultures

in contrast to results obtained from RT-qPCR analysis (Additional file 3: Table S2), suggesting the potential involvement of PgFur in the regulation of processing of gingipains.

\section{Glycosylation of $P$. gingivalis macromolecules}

Microarray analysis suggested differences between the wild-type and mutant strains in the expression of some genes potentially involved in glycosylation processes. As shown in Fig. 7a, one of the selected glycosyltransferases examined was differentially expressed in the mutant strains compared with the wild-type strains, especially under low iron/heme conditions. To visualize potential changes in glycosylation of glycoconjugates produced by $P$. gingivalis, lectin blotting analysis was employed. As shown in Fig. 7 b, significant differences in glycosylation pattern could be observed between A7436 and ATCC 33277 wild-type strains (mostly visible in reactivity with MAL II, AAL, RCA 120, and JACALIN), as well as between growth conditions (mostly visible in reactivity with AAL and SNA). Moreover, we observed changes in glycosylation of glycoconjugates produced by the TO16 mutant strain compared with the ATCC 33277 wild-type strain (mostly visible in reactivity with MAL II, RCA 120 and SNA).

\section{Production of HmuY protein}

Recently, we demonstrated that the TO6 mutant strain produced higher levels of $h m u Y$ transcript [31]. Here we observed a similar tendency in the case of the TO16 mutant strain, although the differences were significantly less pronounced (Additional file 3: Table S2 and Fig. 8 a). In 

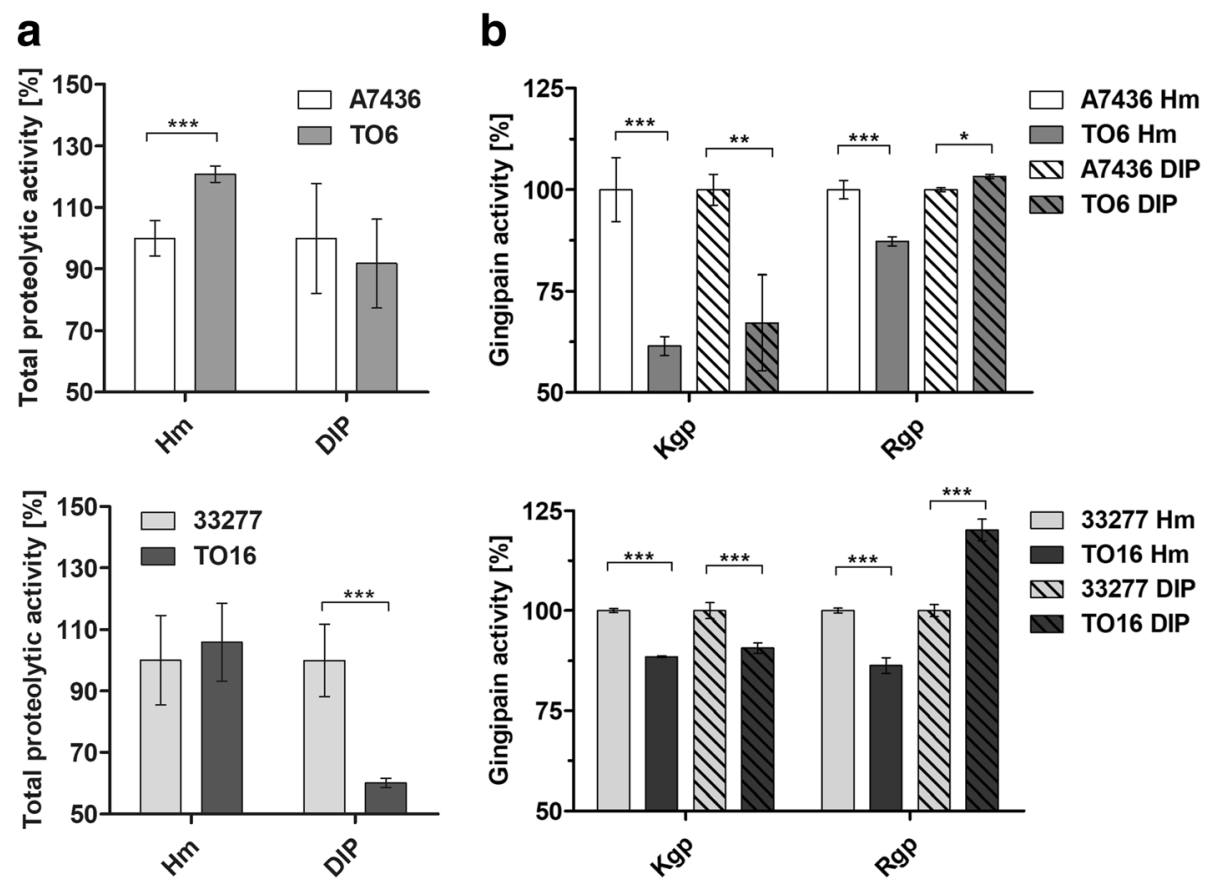

Fig. 5 Total proteolytic (a) and gingipain activities (b) of P. gingivalis wild-type (A7436 and ATCC 33277) and pgfur mutant (TO6 and TO16) strains. Bacteria were grown under high- (Hm) or low-iron/heme (DIP) conditions in liquid culture medium for $24 \mathrm{~h}$ and proteolytic activity of whole cultures against azocasein (a) or protease substrates specific for arginine- (Rgp) or lysine-specific (Kgp) gingipains was determined. ${ }^{*} P<0.05$,

${ }^{* *} P<0.01,{ }^{* * *} P<0.001$

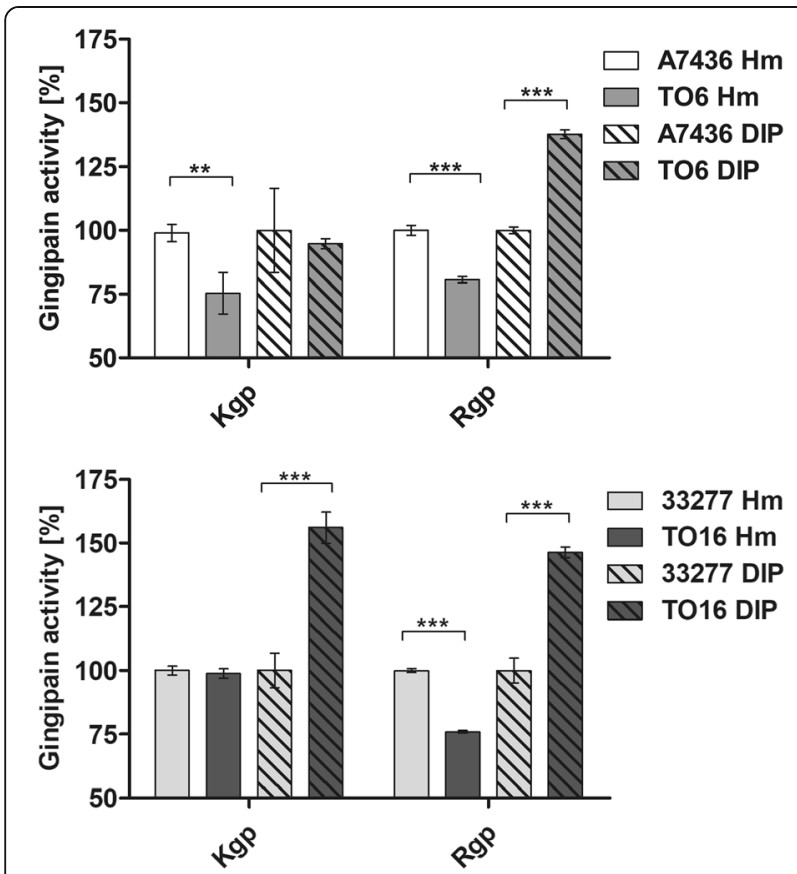

Fig. 6 Gingipain activity of $P$. gingivalis wild-type (A7436 and ATCC 33277) and pgfur mutant (TO6 and TO16) strains. Bacteria were grown under high- $(\mathrm{Hm})$ or low-iron/heme (DIP) conditions in liquid culture medium for $24 \mathrm{~h}$ and gingipain activity in separated culture medium was determined against protease substrates specific for arginine- (Rgp) or lysine-specific (Kgp) gingipains. ${ }^{*} P<0.05$, ${ }^{*} P P<0.01,{ }^{* * *} P<0.001$ addition, a different pattern of mRNA encoding $\mathrm{HmuY}$ protein after inactivation of the pgfur gene could be observed depending on the growth phase (Additional file 3: Table S2 and Fig. 8 a). Inter-strain differences could also be observed in production of the HmuY protein (Fig. 8 b). In general, A7436 and TO6 strains produced higher levels of the HmuY protein, determined in whole bacterial cultures (WC), compared with ATCC 33277 and TO16 strains, all cultured under low-iron/heme conditions. Complementing the lack of the pgfur gene caused partial restoration of the ability of the mutant cells to produce $\mathrm{HmuY}$ protein, as determined in whole bacterial cultures (Additional file 2: Figure S1). Under high-iron/heme conditions $(\mathrm{Hm})$, both mutant strains produced larger amounts of the HmuY proteins compared with their wildtype counterparts (Fig. 8 b). Under iron and heme starvation (DIP), a similar pattern was observed for ATCC 33277 and TO16 strains. Analysis of OMVs demonstrated a similar expression pattern of the HmuY protein compared with the whole cells (WC). When analyzing soluble $\mathrm{HmuY}$ protein shed from the bacterial cells and OMVs and accumulated in the culture medium (CM), the main difference was visible in the $\mathrm{HmuY}$ protein detected in the wild-type strains and mutant strains under iron/hemelimited conditions. TO6 and TO16 mutant strains secreted and accumulated in the culture medium lower amounts of soluble HmuY protein (Fig. 8 b). 


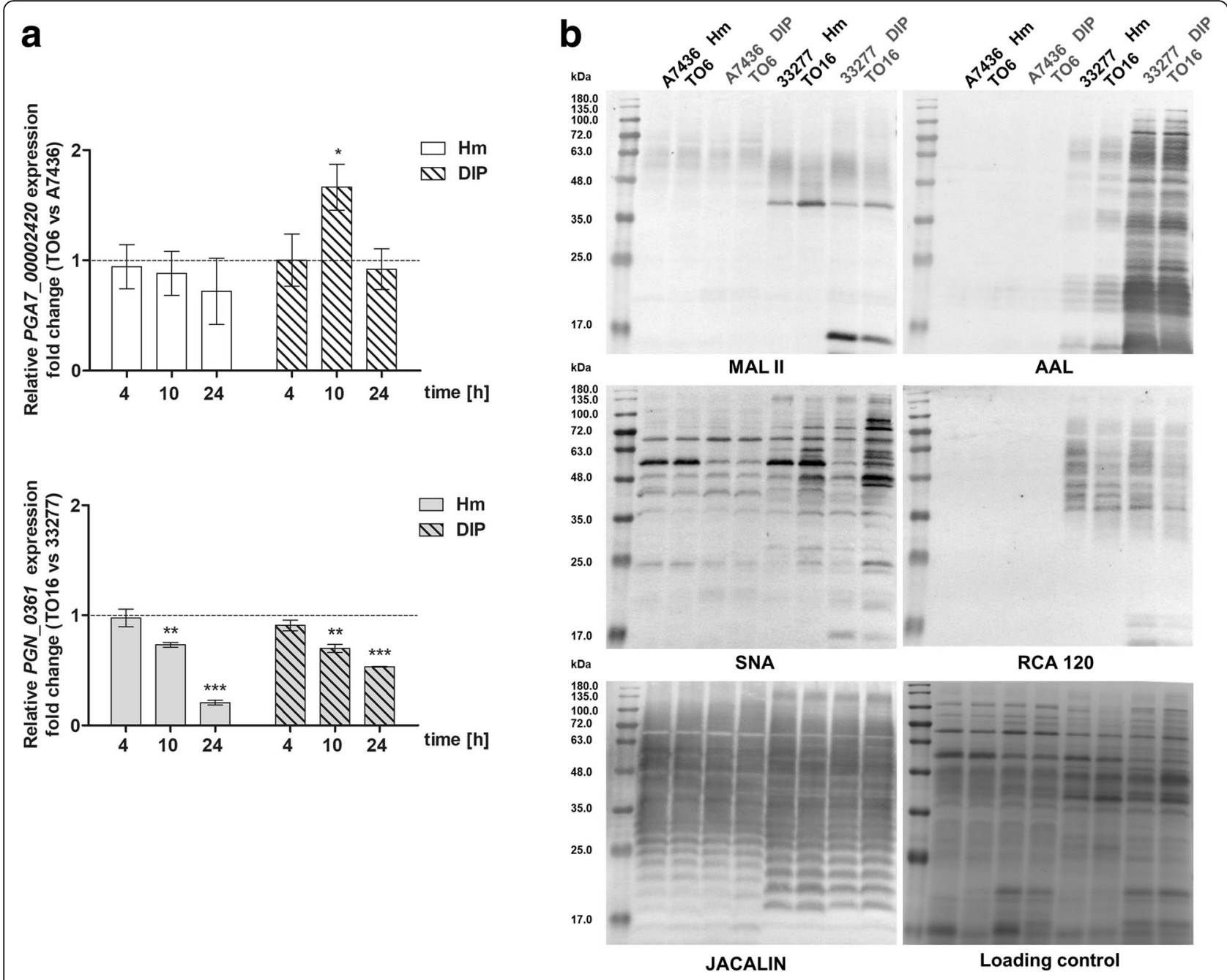

Fig. 7 Analysis of expression of selected glycosyltransferase gene and glycosylation pattern of glycoconjugates produced by $P$. gingivalis wildtype (A7436 and ATCC 33277) and pgfur mutant (TO6 and TO16) strains. Bacteria were grown under high- (Hm) or low-iron/heme (DIP) conditions in liquid culture medium. a Relative gene expression was determined at the indicated time points in pgfur mutant (TO6 and TO16) strains versus wild-type (A7436 and ATCC 33277) strains by RT-qPCR. b Whole bacterial lysates were separated by SDS-PAGE, transferred onto nitrocellulose membranes and probed with selected lectins. MAL II, sialic acid; AAL, fructose linked to $\mathrm{N}$-acetylglucosamine with a-1,6 linkage or to $\mathrm{N}$-acetyllactosamine with a-1,3 linkage, arabinose; SNA, sialic acid linked to the terminal galactose with a-2,6 or a-2,3 linkage; RCA 120, galactose and $N$-acetylgalactosamine; JACALIN, galactose linked to $N$-acetylgalactosamine with $\beta-1,3$ linkage. Equal loading of proteins was visualized in SDSPAGE gels by staining with Coomassie Brilliant Blue G-250 (loading control). ${ }^{*} P<0.05,{ }^{* *} P<0.01$, ${ }^{* * *} P<0.001$

\section{Infection ability of $P$. gingivalis strains against macrophages}

To demonstrate the importance of PgFur for pathogenicity, we analyzed infection ability (adhesion and invasion) and intracellular survival ability of two sets of $P$. gingivalis wild-type and pgfur mutant strains after infection of THP-1-derived macrophages. In general, the most pronounced differences were observed between A7436 and TO6 strains compared with ATCC 33277 and TO16 strains (Fig. 9). In agreement with our previous study [31], P. gingivalis adhesion to the macrophages was lower for the TO6 mutant strain compared with the A7436 wild-type strain (Fig. 9). Surprisingly, no significant differences were observed for the TO16 mutant strain compared with the ATCC 33277 wild-type strain. Importantly, both mutant strains exhibited lower survival ability inside macrophages compared with the wild-type strains, confirming involvement of PgFur in the regulation of expression of genes required for efficient infection.

\section{Discussion}

$P$. gingivalis expresses its transcriptome and proteome in response to environmental changes [19, 31, 38-40]. Cell density and iron/heme availability are important signals affecting the physiology of bacterial cells and are 

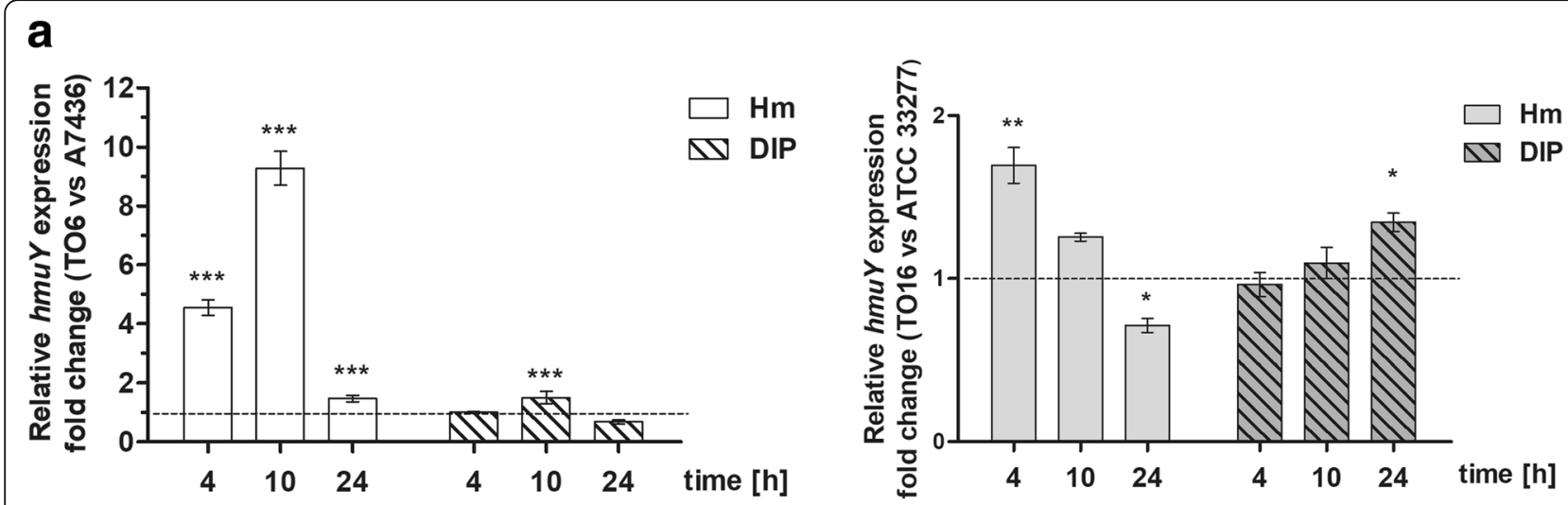

b
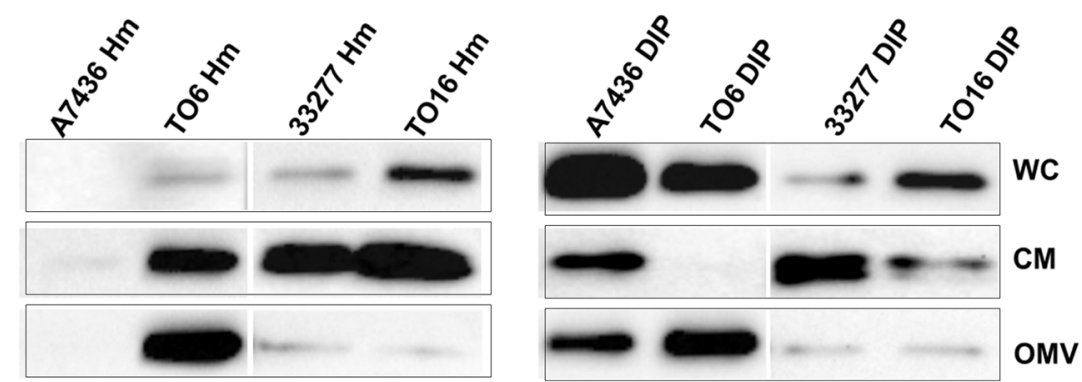

Fig. 8 Expression of $P$. gingivalis hmuY gene. Determination of hmuY transcript was analyzed using RT-qPCR (a) and HmuY protein using Western blotting with anti-HmuY antibodies (b). Bacteria were grown under high- $(\mathrm{Hm})$ or low-iron/heme (DIP) conditions in a liquid culture medium. Relative gene expression was determined in pgfur mutant (TO6 and TO16) strains versus wild-type (A7436 and ATCC 33277) strains. WC, whole bacterial culture; CM, culture medium; OMV, outer-membrane vesicles. ${ }^{*} P<0.05,{ }^{*} P<0.01,{ }^{* * *} P<0.001$

important for the pathogenicity of $P$. gingivalis. However, it has been suggested that some strains may have the capacity to cause dysbiosis and disease, and differences between strains in the ability to cause localized or systemic infections have been observed in animal model studies [41-47]. For example, the ATCC 33277 strain (non-encapsulated) forms higher biofilm structures, is highly fimbriated, but less virulent, whereas the A7436 strain (encapsulated) is less fimbriated, produces smaller biofilm structures, but exhibits higher virulence in animal model studies [48-52]. Several reports have shown that diversity in production of specific virulence factors by $P$. gingivalis might be caused by several mechanisms, including specific domain rearrangements and allelic exchange $[43,47,51,53-56]$. One example is the twocomponent regulatory system composed of the histidine kinase HaeS and the response regulator HaeR. The HaeSR system plays a role in regulation of the $h m u$ operon as well as of genes encoding gingipains [57]. The ATCC 33277 strain lacks HaeS kinase due to the partial deletion of the haeS gene. However, the introduction of this gene into the ATCC 33277 strain allowed observation of the restoration of this system present in the W83 strain, which is highly similar to the A7436 strain. This fact could partly explain the differences in gene expression of the $h m u$ operon and the different amounts of the HmuY protein produced by A7436 and ATCC 33277 strains. This may also enable one to produce different phenotypes by $P$. gingivalis strains, especially those which are more useful for bacterial survival under hostile host conditions, allowing more efficient nutrient uptake and avoidance of the host response.

Recently, we [31] and others [58, 59] demonstrated that PgFur does not regulate iron homeostasis in a manner typical for classical Fur proteins by changes of expression of genes usually involved in this process in other bacteria. Our data [31] are in accordance with the results published by Anaya-Bergman et al. [58] and Butler et al. [59], although regulation of transcription depending on iron and heme binding to the protein and a role in heme-responsive biofilm development have been suggested for the $P$. gingivalis Fur homolog by Butler et al. [60]. We found that expression of other proteins involved in iron and heme acquisition, namely some hemagglutinins, hemolysins and proteases, which are the most important $P$. gingivalis virulence factors, could be regulated by PgFur [31 and this study]. Interestingly, among them are genes encoded by the $h m u$ operon, allowing $P$. gingivalis to efficiently acquire heme [17]. As shown previously [31] and in the present study, 

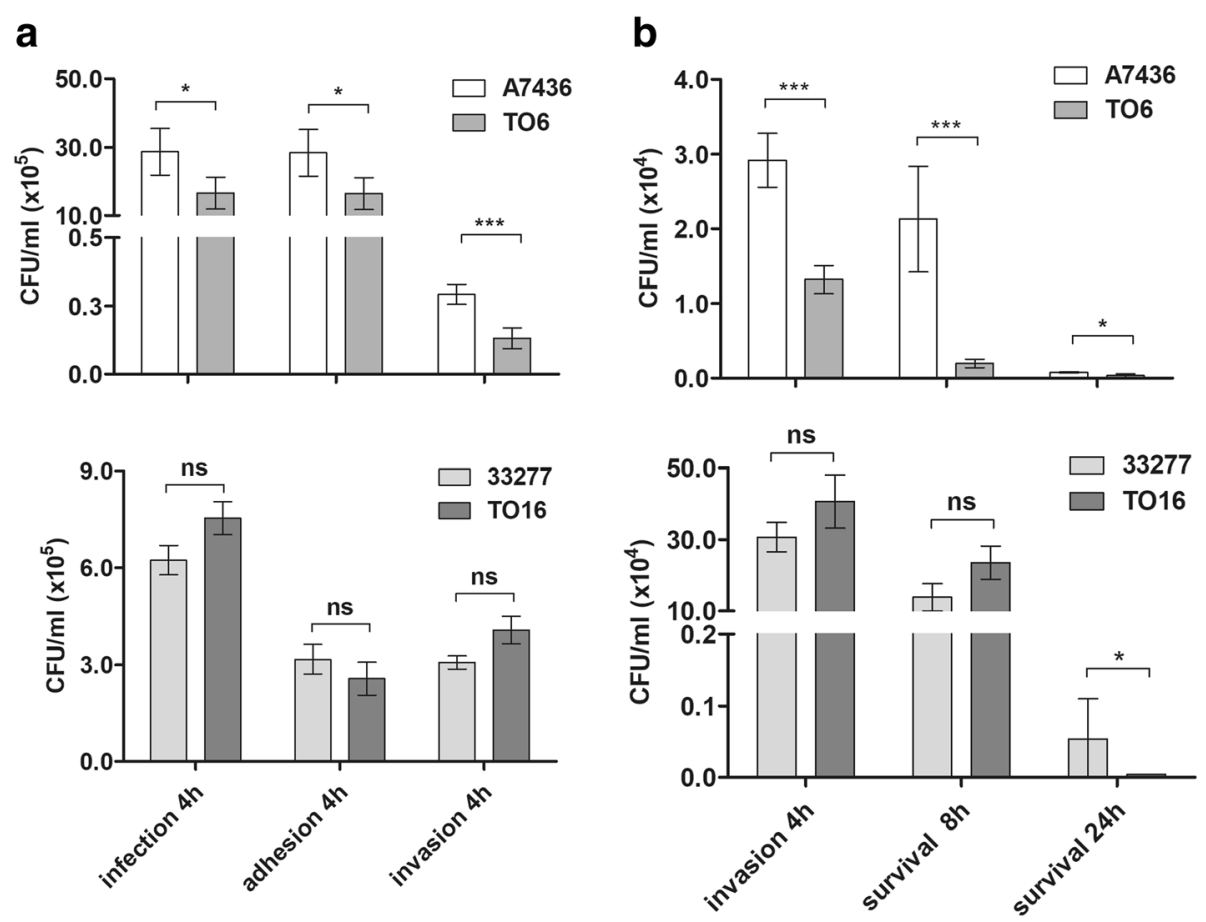

Fig. 9 Infection ability of $P$. gingivalis pgfur mutant strains against human macrophages. a The infection efficiency was determined by counting live bacteria adhering to macrophages (adhesion) and live bacteria invading macrophages (invasion). b Survival ability was determined by counting live bacteria which invaded and survived inside macrophages. ${ }^{*} P<0.05$, ${ }^{* *} P<0.001$, ns - statistically not significant

inactivation of the pgfur gene caused changes in expression of $h m u Y$ gene during $P$. gingivalis growth. Production of higher $h m u Y$ transcript as well as HmuY protein levels during $P$. gingivalis cultivation under rich iron and heme conditions of the TO6 and TO16 mutant strains compared with the wild-type strains suggests that the $h m u$ operon can be regulated by PgFur. It is worth noting at this stage of our studies that $h m u Y$ expression is probably not only regulated by a simple, direct interaction between PgFur and the $h m u Y$ promoter region. We assume that PgFur, as a member of a complex regulatory network, might regulate expression of other regulatory genes, including two-component systems and other transcriptional factors. It was shown before [6164] that Fur protein may influence indirectly gene regulation through proteins from the Crp/Fnr superfamily. A decrease in expression of the two genes encoding putative transcriptional factors from the Crp/Fnr family in both TO6 and TO16 compared with the wild-type strains confirmed a similar relationship between PgFur and proteins encoded by PGA7_00015190/PGN_1569 and PGA7_00004090/PGN_0537 genes in P. gingivalis. However, further studies are required to determine the mutual dependence of these proteins.

In bacterial liquid media $[18,19]$ and inside host cells [16], mRNA encoding $h m u Y$ and HmuY protein are produced with higher yields under low iron/heme conditions. In order to efficiently bind and deliver heme back to the bacterial cell, HmuY is released from the lipid anchor by specific cleavage by Kgp gingipain [19, 21]. We observed smaller amounts of HmuY protein accumulated in the culture medium in the pgfur mutant strains, which might be, at least in part, explained by lower Kgp activity exhibited by cultures of TO6 and TO16 mutant strains. Based on our data, one could assume that such an effect might result from involvement of PgFur in the regulation of processes responsible for gingipain processing and maturation, including glycosylation. Several studies have demonstrated that glycosylation is one of the most important modifications used by $P$. gingivalis to increase its virulence. Bacterial glycosylation is related mainly to macromolecules exposed to the external environment [65] and is important for bacterial interactions with and adhesion to the host cells [66]. Among the best-characterized glycoconjugates are gingipains $[67,68]$, an outer membrane OMP85 protein [69], and Mfa1 fimbriae [70]. Differences in glycosylation observed between the wild-type strains examined in the present study could result from the differences in production of some glycosylated macromolecules in the ATCC 33277 strain [69-71]. It was shown before that changes in glycosylation of macromolecules may influence colony morphology of $P$. gingivalis and other bacteria [72-74]. In agreement with those findings, the 
TO16 mutant strain differed in glycosylation pattern compared with the ATCC 33277 wild-type strain. It can explain, at least in part, the difference in colony shape of the mutant TO16 strain. In addition, some subtle changes were demonstrated between the wild-type and pgfur mutant strains.

We demonstrated that during prolonged culture on a solid medium the TO6 mutant strain formed lightcolored colonies, unlike A7436 and TO16 strains. Lack of pigmentation was reported for $P$. gingivalis strains with dysfunction of some proteins, including those of the type IX secretion system [75-77]. Disturbance in gingipain secretion and maturation may lead to lower ability of the bacteria to accumulate heme on the bacterial cell surface. In addition, this fact might explain the increased expression of the $k g p$ gene at the transcriptional level, especially in the early growth phase of the pgfur mutant strains, despite the decrease in Kgp proteolytic activity. Moreover, we observed discrepancy between enzymatic activity of a total pool of Kgp or Rgp gingipains and their secreted, soluble forms. Similar effects were reported by others in case of $P$. gingivalis mutants with affected gingipain production (e.g., [78, 79]). Our results suggest that multicomponent maturation pathways of gingipains could be affected due to the lack of functional pgfur gene. Therefore, we suggest that PgFur can participate rather indirectly in a regulatory network responsible for gingipain production and activity. Moreover, we believe that the observed dissimilarity in both mutant strains compared with the wild-type strains might be explained, at least in part, by differences in glycosylation and encapsulation of the A7436 strain.

Our recent study [31] showed that the TO6 mutant strain differed in composition of membrane-associated proteins. Together with findings from the present study, including changes in glycosylation between pgfur mutant strains and respective wild-type strains as well as differences between A7436 and ATCC 33277 wild-type strains, we tested interactions between $P$. gingivalis and other periodontopathogens. We found that pgfur gene differently influences the interaction between both wild-type strains of $P$. gingivalis and $P$. intermedia or T. forsythia. This effect might be explained by the difference in composition of the outer membrane of ATCC 33277 and A7436 strains due to the encapsulation of the latter. It is also worth noting that A7436 and ATCC 33277 strains differ in fimbriae production, and the A7436 strain does not produce short Mfa1 fimbriae, composed of glycosylated proteins. This might also, at least in part, explain the higher ability of the ATCC 33277 strain to co-aggregate with S. gordonii compared with the A7436 strain. Our findings are in agreement with data published by Park et al. [80], who demonstrated the importance of Mfa1 fimbriae for synergistic biofilm formation between $P$. gingivalis and $S$. gordonii.
Macrophages belong to essential components of the host immune system involved in the pathogenesis of a variety of inflammatory diseases [81, 82]. Elevated numbers of macrophages with increased levels of proinflammatory cytokines, which significantly contribute to tissue breakdown, were found in samples derived from chronic periodontitis patients [82-84]. It has been demonstrated that $P$. gingivalis can persist in naïve (MØ) and M2-like macrophages, but not in M1-like macrophages for $24 \mathrm{~h}$ [85]. On the other hand, activated M1 macrophages were more prevalent and the M1/M2 ratio was found to be increased in gingival tissues derived from patients with chronic periodontitis [82]. Recently, we demonstrated that the $P$. gingivalis A7436 strain not producing PgFur protein exhibited significantly diminished ability to adhere to host cells, and most importantly to invade HeLa epithelial cells, as well as U937 and THP-1 macrophages, suggesting that this protein is crucial for efficient infection of host cells [31]. In the present study, we observed a difference in the impact of PgFur protein on the interaction of different $P$. gingivalis wild-type strains with macrophages. We believe that the observed differences in outer membrane composition may also be crucial for this process. A difference in production of fimbriae and glycoconjugates may influence the ability of $P$. gingivalis to adhere to and invade macrophages. Another explanation might be a decrease in expression of genes encoding components of the CRISPR/Cas system in pgfur mutant strains compared with the wild-type counterparts. Recently, Heidrich et al. [86] suggested a novel function of the CRISPR/Cas system of Neisseria meningitidis by demonstration of lower adhesion ability of a strain lacking the cas 9 gene. In accordance with those findings, differences observed for mutant strains could be explained by changes in the composition of CRISPR/Cas-associated proteins (e.g., disruption of gene encoding Cas1 protein in the ATCC 33277 strain) and their expression, which results in significant differences observed only in the A7436 strain. As mentioned above, also impairment in pigmentation of the TO6 mutant strain may explain its lower survival ability inside macrophages. Pigmentation was considered one of the major virulence factors of $P$. gingivalis, associated with colonization, maintaining a local anaerobic environment by binding oxygen molecules, and defense against reactive oxygen species produced by immune cells. Data obtained in this study confirmed that bacterial tolerance and the response to oxidative stress might be crucial for $P$. gingivalis survival inside host cells. Based on our results, one may conclude that inactivation of the pgfur gene in A7436 results in significantly lower efficiency to adhere to, invade and survive inside THP-1-derived macrophages. However, compared with the ATCC 33277 strain, the A7436 strain 
is able to survive more efficiently inside macrophages and subsequently spread between the cells, thus increasing its virulence potential.

\section{Conclusions}

In this study, we present for the first time a comparative analysis of pgfur gene inactivation effects in less and more virulent $P$. gingivalis strains. We demonstrate that inactivation of the pgfur gene in the $P$. gingivalis ATCC 33277 strain is reflected in significantly less visible changes in expression of genes encoding potential virulence factors compared with the $P$. gingivalis A7436 strain. Based on our data, one may draw the general conclusion that the two strains demonstrate different phenotypes not only when in the wild-type form but importantly also upon pgfur gene inactivation.

\section{Additional files}

Additional file 1: Table S1. Primers used in this study. (PDF $132 \mathrm{~kb}$ ) Additional file 2: Figure S1. Complementation of pgfur inactivation in $P$. gingivalis mutant strains. Bacteria were grown under high-iron/heme $(\mathrm{BM}+\mathrm{Hm})$ conditions in liquid culture medium for $24 \mathrm{~h}$. Optical density of the bacterial cultures after $24 \mathrm{~h} \mathrm{(a)} \mathrm{and} \mathrm{HmuY} \mathrm{protein} \mathrm{production} \mathrm{in}$ whole cell cultures (b) were determined. A7436, ATCC 33277 - wild-type strains; TO6, TO16 - pgfur mutant strains; TO6 + pgfur, TO16 + pgfur; complemented pgfur mutant strains. ${ }^{* *} P<0.001$, ns - statistically not significant. (PDF 104 kb)

Additional file 3: Table S2. RT-qPCR analysis of expression of selected genes in P. gingivalis pgfur mutant strains (TO6 and TO16) versus wildtype strains (A7436 and ATCC 33277). (PDF $135 \mathrm{~kb}$ )

Additional file 4: Figure S2. Heme binding to whole $P$. gingivalis cells. (PDF $94 \mathrm{~kb}$ )

\section{Acknowledgments}

Prof. Naoya Ohara (Department of Oral Microbiology, Graduate School of Medicine, Dentistry and Pharmaceutical Sciences, Okayama University, Okayama, Japan) is acknowledged for kindly providing us with the pTIO-1 plasmid. Members of the Laboratory of Microbiology (Faculty of Biochemistry, Biophysics and Biotechnology, Jagiellonian University, Krakow, Poland) are acknowledged for replacing ermF cassette with tetQ cassette in the original pTIO-1 plasmid. Richard Ashcroft is kindly acknowledged for the language correction.

\section{Author' contributions}

MŚ, TO conceived the study; MŚ, PS, TO performed the experiments; MŚ, PS, MO, TO analyzed the data; TO, MŚ wrote the draft of the manuscript; MŚ, PS, $\mathrm{MO}, \mathrm{TO}$ revised and approved the final manuscript.

\section{Funding}

This study was supported by grants no. 2014/15/B/NZ6/01723 (TO) and 2014/15/N/NZ6/01718 (MŚ) from National Science Center (NCN, Poland).

\section{Availability of data and materials}

Data generated and analyzed in this study are included in this published article and its supplementary information files or are available upon request.

Ethics approval and consent to participate

Not applicable.

\section{Consent for publication}

Not applicable.

\section{Competing interests}

The authors declare that they have no competing interests.

Received: 3 December 2018 Accepted: 4 June 2019

Published online: 11 June 2019

\section{References}

1. Craig RG, Yip YK, So MK, Boylan RJ, Socransky SS, Haffajee AD. Relationship of destructive periodontal disease to the acute-phase response. J Periodontol. 2003;74:1007-16.

2. Graves DT, Jiang Y, Genco CA. Periodontal disease: bacterial virulence factors, host response and impact on systemic health. Curr Opin Infect Dis. 2000;13:227-32.

3. Socransky SS, Haffajee AD, Cugini MA, Smith C, Kent RL Jr. Microbial complexes in subgingival plaque. J Clin Periodontol. 1998;25:134-44.

4. Griffen AL, Becker MR, Lyons SR, Moeschberger ML, Leys EJ. Prevalence of Porphyromonas gingivalis and periodontal health status. J Clin Microbiol. 1998;36:3239-42.

5. Haffajee AD, Cugini MA, Tanner A, Pollack RP, Smith C, Kent RL Jr, et al. Subgingival microbiota in healthy, well-maintained elder and periodontitis subjects. J Clin Periodontol. 1998;25:346-53.

6. Kolenbrander PE, Andersen RN, Blehert DS, Egland PG, Foster JS, Palmer RJ Jr. Communication among oral bacteria. Microbiol Mol Biol Rev. 2002;66: 486-505.

7. Kamaguchi A, Ohyama T, Sakai E, Nakamura R, Watanabe T, Baba H, et al. Adhesions encoded by the gingipain genes of Porphyromonas gingivalis are responsible for co-aggregation with Prevotella intermedia. Microbiology. 2003;149:1257-64.

8. Bostanci N, Belibasakis GN. Porphyromonas gingivalis: an invasive and evasive opportunistic pathogen. FEMS Microbiol Lett. 2012;333:1-9.

9. Darveau RP, Hajishengallis G, Curtis MA. Porphyromonas gingivalis as a potential community activist for disease. J Dent Res. 2012;91:816-20.

10. Marsh PD. Dental plaque as a microbial biofilm. Caries Res. 2004;38:204-11.

11. Kuboniwa M, Lamont RJ. Subgingival biofilm formation. Periodontol 2000. 2010;52:38-52.

12. Lamont RJ, Chan A, Belton CM, Izutsu KT, Vasel D, Weinberg A. Porphyromonas gingivalis invasion of gingival epithelial cells. Infect Immun. 1995;63:3878-85.

13. Vitkov L, Krautgartner WD, Hannig M. Bacterial internalization in periodontitis. Oral Microbiol Immunol. 2005;20:317-21.

14. Yilmaz O, Verbeke P, Lamont RJ, Ojcius DM. Intercellular spreading of Porphyromonas gingivalis infection in primary gingival epithelial cells. Infect Immun. 2006;74:703-10.

15. Guyodo H, Meuric V, Le Pottier L, Martin B, Faili A, Pers JO, et al. Colocalization of Porphyromonas gingivalis with $\mathrm{CD} 4+\mathrm{T}$ cells in periodontal disease. FEMS Immunol Med Microbiol. 2012;64:175-83.

16. Olczak T, Sosicka P, Olczak M. HmuY is an important virulence factor for Porphyromonas gingivalis growth in the heme-limited host environment and infection of macrophages. Biochem Biophys Res Commun. 2015;467: 748-53.

17. Smalley JW, Olczak T. Haem acquisition mechanisms of Porphyromonas gingivalis - strategies used in polymicrobial community in a haem-limited host environment. Mol Oral Microbiol. 2017;32:1-23.

18. Olczak T, Sroka A, Potempa J, Olczak M. Porphyromonas gingivalis HmuY and HmuR: further characterization of a novel mechanism of heme utilization. Arch Microbiol. 2008;183:197-210.

19. Olczak T, Wojtowicz H, Ciuraszkiewicz J, Olczak M. Species specificity, surface exposure, protein expression, immunogenicity, and participation in biofilm formation of Porphyromonas gingivalis HmuY. BMC Microbiol. 2010;10:134.

20. Veith PD, Chen YY, Gorasia DG, Chen D, Glew MD, O'Brien-Simpson NM, et al. Porphyromonas gingivalis outer membrane vesicles exclusively contain outer membrane and periplasmic proteins and carry a cargo enriched with virulence factors. J Proteome Res. 2014;13:2420-32.

21. Wojtowicz H, Guevara T, Tallant C, Olczak M, Sroka A, Potempa J, et al. Unique structure and stability of HmuY, a novel heme-binding protein of Porphyromonas gingivalis. PLoS Pathog. 2009:5(5):e1000419.

22. Potempa J, Sroka A, Imamura T, Travis J. Gingipains, the major cysteine proteinases and virulence factors of Porphyromonas gingivalis: structure, function and assembly of multidomain protein complexes. Curr Protein Pept Sci. 2003;4:397-407. 
23. Guo Y, Nguyen KA, Potempa J. Dichotomy of gingipains action as virulence factors: from cleaving substrates with the precision of a surgeon's knife to a meat chopper-like brutal degradation of proteins. Periodontol 2000. 2010; 54:15-44.

24. Hantke K. Iron and metal regulation in bacteria. Curr Opin Microbiol. 2001;4: 172-7.

25. Troxell B, Hassan HM. Transcriptional regulation by ferric uptake regulator (Fur) in pathogenic bacteria. Front Cell Infect Microbiol. 2013;3:59.

26. Fillat MF. The FUR (ferric uptake regulator) superfamily: diversity and versatility of key transcriptional regulators. Arch Biochem Biophys. 2014;546: 41-52.

27. Dubrac S, Touati D. Fur positive regulation of iron superoxide dismutase in Escherichia coli: functional analysis of the $\operatorname{sodB}$ promoter. J Bacteriol. 2000; 182:3802-8.

28. Delany I, leva R, Alaimo C, Rappuoli R, Scarlato V. The iron-responsive regulator Fur is transcriptionally autoregulated and not essential in Neisseria meningitides. J Bacteriol. 2001;185:6032-41.

29. Hernandez JA, Peleato ML, Fillat MF, Bes MT. Heme binds to and inhibits the DNA-binding activity of the global regulator FurA from Anabaena sp. PCC 7120. FEBS Lett. 2004;577:35-41.

30. Lee JW, Helmann JD. Functional specialization within the Fur family of metalloregulators. Biometals. 2007;20:485-99.

31. Ciuraszkiewicz J, Smiga M, Mackiewicz P, Gmiterek A, Bielecki M, Olczak M, et al. Fur homolog regulates Porphyromonas gingivalis virulence under lowiron/heme conditions through a complex regulatory network. Mol Oral Microbiol. 2014:29:333-53.

32. Tagawa J, Inoue T, Naito M, Sato K, Kuwahara T, Nakayama M, et al. Development of a novel plasmid vector pTIO-1 adapted for electrotransformation of Porphyromonas gingivalis. J Microbiol Methods. 2014:105:174-9.

33. Smiga M, Bielecki M, Olczak M, Smalley JW, Olczak T. Anti-HmuY antibodies specifically recognize Porphyromonas gingivalis HmuY protein but not homologous proteins in other periodontopathogens. PLoS One. 2015;10(2): e0117508.

34. Pfaffl MM. A new mathematical model for relative quantification in real-time RT-PCR. Nucleic Acids Res. 2001;29(9):e45.

35. Cronan CA, Potempa J, Travis J, Mayo JA. Inhibition of Porphyromonas gingivalis proteinases (gingipains) by chlorhexidine: synergistic effect of Zn(II). Oral Microbiol Immunol. 2006;21:212-7.

36. Gmiterek A, Klopot A, Wojtowicz H, Trindade SC, Olczak M, Olczak T. Immune response of macrophages induced by Porphyromonas gingivalis requires HmuY protein. Immunobiol. 2016;221:1382-94.

37. Pinochet-Barros A, Helmann JD. Redox sensing by $\mathrm{Fe}^{2+}$ in bacterial Fur family metalloregulators. Antioxid Redox Signal. 2018;29:1858-71.

38. Xie H, Cai S, Lamont RJ. Environmental regulation of fimbrial gene expression in Porphyromonas gingivalis. Infect Immun. 1997;65:2265-71.

39. Hirano T, Beck DA, Demuth DR, Hackett M, Lamont RJ. Deep sequencing of Porphyromonas gingivalis and comparative transcriptome analysis of a LuxS mutant. Front Cell Infect Microbiol. 2012;2:79.

40. Hovik H, Yu WH, Olsen I, Chen T. Comprehensive transcriptome analysis of the periodontopathogenic bacterium Porphyromonas gingivalis W83. J Bacteriol. 2012;194:100-14.

41. Griffen AL, Lyons SR, Becker MR, Moeschberger ML, Leys EJ. Porphyromonas gingivalis strain variability and periodontitis. J Clin Microbiol. 1999;37:4028-33.

42. Gui MJ, Dashper SG, Slakeski N, Chen YY, Reynolds EC. Spheres of influence: Porphyromonas gingivalis outer membrane vesicles. Mol Oral Microbiol. 2016;31:365-78

43. Imai M, Murakami Y, Nagano K, Nakamura H, Yoshimura F. Major outer membrane proteins from Porphyromonas gingivalis: strain variation, distribution, and clinical significance in periradicular lesions. Eur J Oral Sci. 2005;113:391-9.

44. Nakagawa I, Inaba H, Yamamura T, Kato T, Kawai S, Ooshima T, et al Invasion of epithelial cells and proteolysis of cellular focal adhesion components by distinct types of Porphyromonas gingivalis fimbriae. Infect Immun. 2006;74:3773-82.

45. Quirynen M, Papaioannou W, van Steenbergen TJ, Dierickx K, Cassiman JJ, van Steenberghe D. Adhesion of Porphyromonas gingivalis strains to cultured epithelial cells from patients with a history of chronic adult periodontitis or from patients less susceptible to periodontitis. J Periodontol. 2001;72:626-33.
46. Rangarajan M, Aduse-Opoku J, Paramonov NA, Hashim A, Curtis MA. Hemin binding by Porphyromonas gingivalis strains is dependent on the presence of A-LPS. Mol Oral Microbiol. 2017;32:365-74.

47. Teixeira SRL, D'Epiro TTS, Pinheiro ET, Simionato MRL, Taniwaki NN, Kisielius $J$, et al. Lineage variability in surface components expression within Porphyromonas gingivalis. Microb Pathog. 2014;77:100-4.

48. Brunner J, Scheres N, El Idrissi NB, Deng DM, Laine ML, van Winkelhoff AJ, et al. The capsule of Porphyromonas gingivalis reduces the immune response of human gingival fibroblasts. BMC Microbiol. 2010;10:5.

49. Laine ML, van Winkelhoff AJ. Virulence of six capsular serotypes of Porphyromonas gingivalis in a mouse model. Oral Microbiol Immunol. 1998; 13:322-5.

50. Umeda JE, Missailidis C, Longo PL, Anzai D, Wikstrom M, Mayer MPA. Adhesion and invasion to epithelial cells by fimA genotypes of Porphyromonas gingivalis. Oral Microbiol Imunol. 2006;21:415-9.

51. Igboin CO, Griffen AL, Leys EJ. Porphyromonas gingivalis strain diversity. J Clin Microbiol. 2009:47:3073-81.

52. Tachibana-Ono M, Yoshida A, Kataoka S, Ansai T, Shintani Y, Takahashi Y, et al. Identification of the genes associated with a virulent strain of Porphyromonas gingivalis using the subtractive hybridization technique. Oral Microbiol Immunol. 2008;23:84-7.

53. Dashper SG, Mitchell HL, Seers CA, Gladman SL, Seemann T, Bulach DM, et al. Porphyromonas gingivalis uses specific domain rearrangements and allelic exchange to generate diversity in surface virulence factors. Front Microbiol. 2017:8:48.

54. Acuna-Amador L, Primot A, Cadieu E, Roulet A, Barloy-Hubler F. Genomic repeats, misassembly and reannotation: a case study with long-read resequencing of Porphyromonas gingivalis reference strains. BMC Genomics. 2018;19:54.

55. Naito M, Hirakawa H, Yamashita A, Ohara N, Shoji M, Yukitake $H$, et al. Determination of the genome sequence of Porphyromonas gingivalis strain ATCC 33277 and genomic comparison with strain W83 revealed extensive genome rearrangements in P. gingivalis. DNA Res. 2008;15:215-25.

56. Chen $\mathrm{T}$, Siddiqui $\mathrm{H}$, Olsen I. In silico comparison of 19 Porphyromonas gingivalis strains in genomics. Phylogenetics, phylogenomics and functional genomics. Front Cell Infect Microbiol. 2017:7-28.

57. Scott JC, Klein BA, Duran-Pinedo A, Hu L, Duncan MJ. A two-component system regulates hemin acquisition in Porphyromonas gingivalis. PLoS One. 2013;8(9):e73351.

58. Anaya-Bergman C, Rosato A, Lewis JP. Iron- and hemin-dependent gene expression of Porphyromonas gingivalis. Mol Oral Microbiol. 2015;30:39-61.

59. Butler C, Mitchell H, Dashper S, Reynolds E. The Porphyromonas gingivalis ferric uptake regulator orthologue does not regulate iron homeostasis. Genomics Data. 2015;5:167-8.

60. Butler CA, Dashper SG, Zhang L, Seers CA, Mitchell HL, Catmull DV, et al The Porphyromonas gingivalis ferric uptake regulator orthologue binds hemin and regulates hemin-responsive biofilm development. PLoS One. 2014;9(11):e111168.

61. Grifantini R, Sebastian S, Frigimelica E, Draghi M, Bartolini E, Muzzi A, et al. Identification of iron-activated and -repressed Fur-dependent genes by transcriptome analysis of Neisseria meningitidis group B. Proc Natl Acad Sc U S A. 2003;100:9542-7.

62. Hassan HM, Sun HC. Regulatory roles of Fnr, Fur, and arc in expression of manganese-containing superoxide dismutase in Escherichia coli. Proc Natl Acad Sci U S A. 1992:89:3217-21.

63. Yang XW, He Y, Xu J, Xiao X, Wang FP. The regulatory role of ferric uptake regulator (Fur) during anaerobic respiration of Shewanella piezotolerans WP3. PLoS One. 2013;8(10):e75588.

64. Yu C, Genco CA. Fur-mediated global regulatory circuits in pathogenic Neisseria species. J Bacteriol. 2012;194:6372-81.

65. Nothaft H, Szymanski CM. Protein glycosylation in bacteria: sweeter than ever. Nat Rev Microbiol. 2010;8:765-78.

66. Li H, Debowski AW, Liao T, Tang H, Nilsson HO, Marshall BJ, et al. Understanding protein glycosylation pathways in bacteria. Future Microbiol. 2017;12:59-72.

67. Gallagher A, Aduse-Opoku J, Rangarajan M, Slaney JM, Curtis MA. Glycosylation of the Arg-gingipains of Porphyromonas gingivalis and comparison with glycoconjugate structure and synthesis in other bacteria. Curr Prot Pept Sci. 2003:4:427-41.

68. Paramonov N, Rangarajan M, Hashim A, Gallagher A, Aduse-Opoku J, Slaney JM, et al. Structural analysis of a novel anionic polysaccharide from 
Porphyromonas gingivalis strain W50 related to Arg-gingipain glycans. Mol Microbiol. 2005;58:847-63.

69. Nakao R, Tashiro Y, Nomura N, Kosono S, Ochiai K, Yonezawa H, et al. Glycosylation of the OMP85 holmolog of Porphyromonas gingivalis and its involvement in biofilm formation. Biochem Biophys Res Commun. 2008;365: 784-9.

70. Zeituni AE, McCaig W, Scisci E, Thanassi DG, Cutler CW. The native 67kilodalton minor fimbria of Porphyromonas gingivalis is a novel glycoprotein with DC-SIGN-targeting motifs. J Bacteriol. 2010;192:4103-10.

71. Kishi M, Hasegawa Y, Nagano K, Nakamura H, Murakami Y, Yoshimura F. Identification and characterization of novel glycoproteins involved in growth and biofilm formation by Porphyromonas gingivalis. Mol Microbiol. 2012;27:458-70.

72. Díaz L, Hoare A, Soto C, Bugueño I, Silva N, Dutzan N, et al. Changes in lipopolysaccharide profile of Porphyromonas gingivalis clinical isolates correlate with changes in colony morphology and polymyxin B resistance. Anaerobe. 2015;33:25-32.

73. Hickman JW, Tifrea DF, Harwood CS. A chemosensory system that regulates biofilm formation through modulation of cyclic diguanylate levels. Proc Natl Acad Sci U S A. 2005;102:14422-7.

74. Ueda A, Wood TK. Connecting quorum sensing, c-di-GMP, pel polysaccharide, and biofilm formation in Pseudomonas aeruginosa through tyrosine phosphatase TpbA (PA3885). PLoS Pathog. 2009;5(6):e1000483.

75. Sato K, Sakai E, Veith PD, Shoji M, Kikuchi Y, Yukitake H, et al. Identification of a new membrane-associated protein that influences transport/maturation of gingipains and adhesins of Porphyromonas gingivalis. J Biol Chem. 2005; 280:8668-77.

76. Sato K, Naito M, Yukitake H, Hirakawa H, Shoji M, McBride MJ, et al. A protein secretion system linked to bacteroidete gliding motility and pathogenesis. Proc Natl Acad Sci U S A. 2010;107:276-81.

77. Veith PD, Glew MD, Gorasia DG, Reynolds EC. Type IX secretion: the generation of bacterial cell surface coatings involved in virulence, gliding motility and the degradation of complex biopolymers. Mol Microbiol. 2017; 106:35-53.

78. Hasegawa Y, Nishiyama S, Nishikawa K, Kadowaki T, Yamamoto K, Noguchi T, Yoshimura F. A novel type of two-component regulatory system affecting gingipains in Porphyromonas gingivalis. Microbiol Immunol. 2003;47:849-58.

79. Vanterpool E, Roy F, Sandberg L, Fletcher HM. Altered gingipain maturation in vimA- and vimE-defective isogenic mutants of Porphyromonas gingivalis. Infect Immun. 2005;73:1357-66.

80. Park Y, Simionato MR, Sekiya K, Murakami Y, James D, Chen W, et al. Short fimbriae of Porphyromonas gingivalis and their role in coadhesion with Streptococcus gordonii. Infect Immun. 2005;73:3983-9.

81. Ebersole JL, Dawson D $3^{\text {rd }}$, Emecen-Huja P, Nagarajan R, Howard K, Grady $M E$, et al. The periodontal war: microbes and immunity. Periodontol 2000. 2017;75:52-115.

82. Zhou LN, Bi CS, Gao LN, An Y, Chen F, Chen FM. Macrophage polarization in human gingival tissue in response to periodontal disease. Oral Dis. 2018. https://doi.org/10.1111/odi.12983.

83. Charon J, Toto PD, Gargiulo AW. Activated macrophages in human periodontitis. J Periodontol. 1981;52:328-35.

84. Seguier S, Gogly B, Bodineau A, Godeau G, Brousse N. Is collagen breakdown during periodontitis linked to inflammatory cells and expression of matrix metalloproteinases and tissue inhibitors of metalloproteinases in human gingival tissue ? J Periodontol. 2001;72:1398-406.

85. Lam RS, O'Brien-Simpson NM, Holden JA, Lenzo JC, Fong SB, Reynolds EC. Unprimed, M1 and M2 macrophages differentially interact with Porphyromonas gingivalis. PLoS One. 2016;11(7):e0158629.

86. Heidrich N, Hagmann A, Bauriedl S, Vogel J, Schoen C. The CRISPR/Cas system in Neisseria meningitidis affects bacterial adhesion to human nasopharyngeal epithelial cells. RNA Biol. 2018;30:1-7.

\section{Publisher's Note}

Springer Nature remains neutral with regard to jurisdictional claims in published maps and institutional affiliations.

Ready to submit your research? Choose BMC and benefit from:

- fast, convenient online submission

- thorough peer review by experienced researchers in your field

- rapid publication on acceptance

- support for research data, including large and complex data types

- gold Open Access which fosters wider collaboration and increased citations

- maximum visibility for your research: over $100 \mathrm{M}$ website views per year

At BMC, research is always in progress.

Learn more biomedcentral.com/submissions 